\title{
Humidity affects the viscoelastic properties of supramolecular living polymers
}

\author{
A. Louhichi and A. R. Jacob \\ Institute of Electronic Structure and Laser, FORTH, Heraklion 70013, Crete, Greece and Department of Materials \\ Science and Technology, University of Crete, Heraklion 71003, Crete, Greece
}

L. Bouteiller

Sorbonne Universités, UPMC Univ. Paris 06, CNRS, Institut Parisien de Chimie Moléculaire, Equipe Chimie des Polymères, 4 Place Jussieu, F-75005 Paris, France

D. Vlassopoulos ${ }^{\text {a) }}$

Institute of Electronic Structure and Laser, FORTH, Heraklion 70013, Crete, Greece and Department of Materials Science and Technology, University of Crete, Heraklion 71003, Crete, Greece

(Received 7 May 2017; final revision received 15 July 2017; published 1 November 2017)

\begin{abstract}
We investigate the linear and nonlinear viscoelastic response of a supramolecular gelator formed by association of a bisurea monomer (EHUT) via hydrogen bonds, in a nonpolar organic solvent (dodecane). Past experimental studies reported contradictory results concerning the concentration dependence and the magnitude of the terminal relaxation time. The discrepancies among data were attributed to the presence of water and, in particular, its chain stopper effects on the EHUT supramolecular assembly. Here, we present new measurements with this system under conditions of controlled humidity, achieved by means of a simple setup developed for this purpose. We resolve the discrepancy and demonstrate that humidity (both during sample storage and measurement) can substantially affect the linear viscoelastic response (frequency spectra). The magnitude of the terminal relaxation time, $\tau_{t}$, is significantly reduced (by as much as 1 order of magnitude) and its concentration dependence follows $\tau_{t} \sim c^{0.77 \pm 0.06}$. This scaling differs from the theoretical prediction with nearly double exponent (1.25). We tentatively attribute this difference to the polydispersity in the supramolecular chain length, which results mainly from the EHUT self-assembly association mechanism, and propose that constraint release effects of smaller chains should be considered as they may weaken the concentration dependence of the terminal relaxation. On the other hand, the magnitude of the plateau modulus $G_{0}$ is affected by humidity to a lesser degree (not exceeding 50\%), whereas its concentration dependence is in accordance with predictions, $G_{0} \sim c^{2 \pm 0.21}$. Finally, the nonlinear viscoelastic properties are also affected, again experiencing weakening in the presence of humidity. C 2017 The Society of Rheology. [http://dx.doi.org/10.1122/1.4997600]
\end{abstract}

\section{INTRODUCTION}

Adding small amounts of organogelator to an organic, nonpolar liquid is known to result in a sharp increase in its zero-shear viscosity and eventually to its gelation [1]. Such a variation in macroscopic properties is particularly important not only scientifically but also technologically, as it finds applications in a wide range of products and processes such as coatings, cosmetics, and oil recovery. The main prerequisite is the synthesis of appropriate low molecular weight compounds [1,2]. A large variety of chemical structures has been reported in the literature, bearing organogelator features. Focusing on such molecules that self-assemble into fibrillar aggregates, the gelation may be understood by invoking the entanglement of fibrils leading and the formation of a network [3-6]. The self-assembly of organogelators is driven by their propensity to form intermolecular H-bonds

\footnotetext{
a) Author to whom correspondence should be addressed; electronic mail: dvlasso@iesl.forth.gr

(C) 2017 by The Society of Rheology, Inc.

and/or develop other specific interactions such as dipolar associations. When each small molecule is linked to its neighbors by such noncovalent reversible bonds, the resulting chains may be viewed as "living polymers," akin to wormlike surfactant micelles (WLM) [7,8]; hence, they can undergo scission and recombination mechanisms which affect the overall dynamics of the system [7,9].

A much investigated system that serves as an archetype is the reversible supramolecular polymer based on a bis-urea moiety (EHUT). Its synthesis, thermodynamic, and some rheological properties have been already reported in the literature [5,10-22]. EHUT forms long cylindrical filaments in nonpolar solvents [20], giving rise to strong viscoelastic response. In fact, these aggregates are reported to be equilibrium structures [5], contrary to those formed by most organogelators, which are metastable, i.e., they keep building up their structure with time [23-25].

Figure 1 represents the phase diagram of EHUT solutions in toluene where the transition between monomers and supramolecular filaments was determined by isothermal titration calorimetry (ITC) and the transition between filaments 
and tubes was determined by ITC, viscometry, fourier transform infrared spectroscopy, and differential scanning calorimetry. In this work, we chose to work in the "tube" region from the phase diagram at a temperature of $25^{\circ} \mathrm{C}$, over a concentration range from 2 to $12 \mathrm{~g} / \mathrm{l}$, where the tubes are long enough to entangle, and exhibiting rich viscoelastic response.

The above diagram has formed the basis for extensive rheological investigations of EHUT supramolecular assemblies in different apolar solvents and regimes over the last 15 years [5,10,12,14,17-22]. The linear viscoelastic properties of the EHUT supramolecular solution were discussed in the framework of the classic theory of Cates for living polymers $[7,8,26]$. The key element of this theory is the fact that worms are characterized by two relaxation times, the local breaking time $\left(\tau_{b}\right)$ due to the exchange of associating units (lifetime of bonds) and the longer terminal relaxation time $\left(\tau_{t}\right)$ of the whole WLM. The former is associated with the plateau modulus of the physical network, whereas the latter with the terminal crossover of the storage and loss moduli, following a single Maxwell behavior at low frequencies. In this work, we define the fast time as the inverse of the frequency at the minimum of $G^{\prime \prime}[27,28]$, which is assigned to the maximum value of the breaking (and reformation) time $\left(\tau_{b}\right)$ of the supramolecular structure. On the other hand, the slow terminal relaxation time $\left(\tau_{t}\right)$ is taken as the inverse of the lowfrequency crossover $\left(\tau_{t}=1 / \omega_{c}\right)$. These times are linked via $\tau_{t}=\left(\tau_{\text {rep }} \tau_{b}\right)^{1 / 2}$, where $\tau_{\text {rep }}$ is the reptation time of the living assembly (this expression holds in the limit $\tau_{b} \ll \tau_{\text {rep }}$ ). They scale with the average micellar length $L$ as $\tau_{b} \sim L^{-1}$ and, based on the classic scaling of the reptation time with concentration in good solvent conditions [29,30]

$$
\tau_{\text {rep }} \sim L^{3} c^{3 / 2}
$$

By further assuming that $L \sim c^{1 / 2}[7]$, which is a direct consequence of the Flory-Huggins mean field theory, one can rewrite the concentration scaling of the terminal relaxation time as

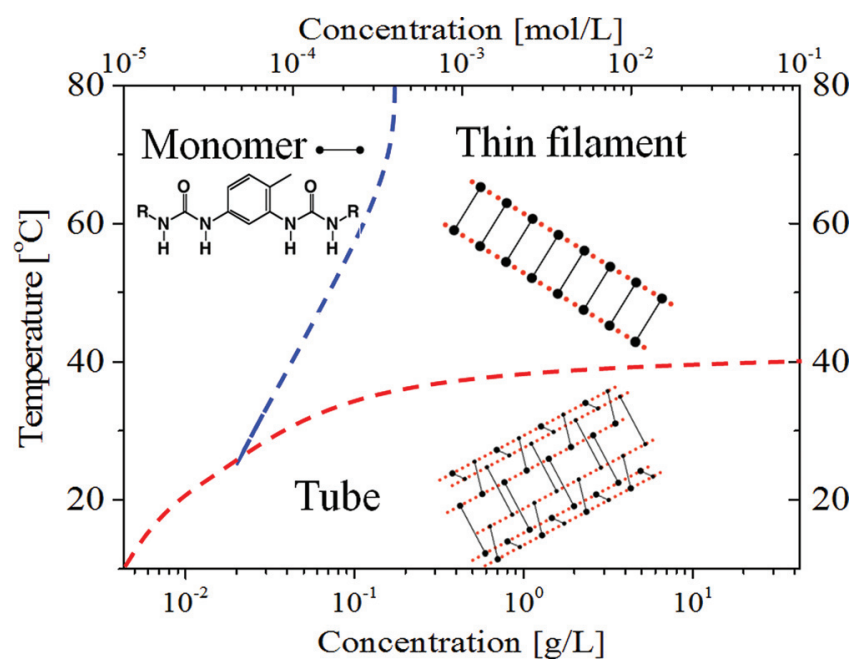

FIG. 1. Phase diagram for EHUT solutions in toluene showing the transition between monomers and supramolecular filaments. The structure of the EHUT monomer is presented, along with a schematic illustration of the various aggregates formed (out of scale), i.e., filaments and tubes [11]. Hydrogen bonds are represented by dotted lines connecting the urea functions (black circles).

$$
\tau_{t} \sim c^{1.25}
$$

In addition to that and by analogy with semidilute polymer solutions in good solvent, Cates and Candau [8] proposed a scaling of the plateau modulus with concentration in WLMs

$$
G \sim c^{2.25}
$$

Concerning the dependence of the terminal relaxation time on $L$, this relies on the estimation of the former. Larson [31] reviewed different approaches to calculate the average chain length from experimental linear viscoelastic data. He suggested that the most accurate way to estimate the average length of the micelles is based on their reptation time. However, the relation of the average micellar length to the reptation time was based on the data with one WLM system with constant length at a single concentration. Nevertheless, by using the scaling in Eq. (1) and by further considering the scaling of the breaking time $\tau_{b} \sim L^{-1}$, the terminal relaxation time [7] is found to scale as $\tau_{t} \sim L^{1}$.

Based on a compilation of various datasets with living polymers [32], the dependence of the plateau modulus on concentration was found to follow a scaling with an exponent that varied from 1.8 to 2.4 , in reasonable agreement with the theoretical prediction in Eq. (3). However, the scaling of the terminal relaxation time in Eq. (2) has not yet been confirmed experimentally. Indeed, the results from different WLMs indicate scaling exponents ranging from negative to positive values, e.g., -1.4 [33] to 1.02 [34]. This large variability of response brought into question the scaling of the average micellar length with concentration. It was suggested that different WLMs may exhibit different scaling [32].

For the EHUT supramolecular system investigated in this work, besides the above-mentioned issue, discrepancies in both the magnitude and the concentration scaling of $\tau_{t}$ have been reported. A scaling exponent of 1.5 can be extracted from the data of van der Gucht et al. in cyclohexane in [17]. Ducouret et al. in [5] used dodecane and reported a value of 0.77 . However, this value is only indicative due to the surprisingly strong scattering of the extracted $\tau_{t}$ data at different concentrations. On the other hand, Shikata et al. in [12] reported a dual scaling for $\tau_{t}$ with concentration in dodecane: In the same concentration range probed by Ducouret et al. in [5], $\tau_{t}$ was independent of concentration, whereas at higher concentrations $\tau_{t} \sim c^{l}$.

Given that EHUT is highly hygroscopic and can form hydrogen bonds, we suspect that humidity is primarily responsible for the observed discrepancies, since this parameter was not controlled in earlier investigations. Here, we present a systematic investigation of the linear and some nonlinear viscoelastic properties of EHUT in dodecane over a wide range of concentrations, under humid and dry conditions, in an attempt to resolve the above-mentioned controversies and explore the potential effects of humidity on terminal relaxation and plateau modulus. In order to achieve this goal, we have built a small drying chamber and adapted it to the rheometer. This has allowed conducting experiments at conditions of well-controlled humidity (typically, the relative humidity was kept below $5 \%$ for $16 \mathrm{~h}$ ). We compare our 
humidity-free experimental results to the theoretical predictions in Eqs. (2) and (3) and show that even a small degree of humidity affects substantially the magnitude of the terminal relaxation time, whereas the modulus is noticeably affected only when the degree of humidity is high. Nonlinear rheology is also found to be very sensitive to humidity. We attribute the differences in the concentration scaling of the terminal relaxation time to polydispersity effects. The manuscript is organized as follows: After the introduction, we present in Sec. II the experimental aspects of the work (sample preparation protocols, humidity control, and rheological measurements). Then, in Sec. III, we present and discuss the experimental results in humid and dry conditions and focus on the concentration dependence of the terminal time and plateau modulus. Finally, the main conclusions are summarized in Sec. IV.

\section{EXPERIMENTAL}

\section{A. Sample preparation}

The EHUT powder was synthetized according to [6]. We used two distinct preparation protocols: Protocol-1 consisted of using the EHUT powder after storage at room temperate in a vial, without any drying. Protocol-2 involved drying the EHUT powder before use, under vacuum and temperature of $50^{\circ} \mathrm{C}$ for about $18 \mathrm{~h}$, in order to remove any trace of humidity absorbed during storage. Subsequently, the desired amount of EHUT powder was immediately added to the desired volume of dodecane and left under stirring at $80^{\circ} \mathrm{C}$ for $48 \mathrm{~h}$. The final solution was completely transparent and thermodynamically stable at room temperature (in fact, there was no sign of precipitation or phase separation in the final solutions for more than a year at $25^{\circ} \mathrm{C}$ ). Note that at this temperature, we are well within the regime of tubes in the concentration range explored (see Fig. 1). Solutions were measured immediately after the end of the preparation protocol.

\section{B. Rheology and humidity-control}

Dynamic viscoelastic measurements were performed with a sensitive strain-controlled ARES-rheometer (100FRTN1; TA Instruments, USA) equipped with a stainless steel coneand-plate geometry of $25 \mathrm{~mm}$ radius and $0.04 \mathrm{rad}$ cone angle. The linear viscoelastic spectra were obtained by applying a small-amplitude sinusoidal strain with varying angular frequency sweep, $\omega$, from 100 to $0.01 \mathrm{rad} / \mathrm{s}$, and detecting the storage and loss moduli, $G^{\prime}$ and $G^{\prime \prime}$, respectively. The linear viscoelastic regime was established by means of dynamic strain sweep tests at different frequencies (typically varying the strain amplitude from $0.1 \%$ to $100 \%$ ), whereas the time stability of the samples before the frequency sweep tests was ensured via linear dynamic time sweeps at $1 \mathrm{rad} / \mathrm{s}$. The nonlinear rheological response was investigated by means of startup experiments in steady shear (at different rates) until steady state was reached. All experiments were performed at $25^{\circ} \mathrm{C}$, achieved by means of a Peltier temperature control system. In order to measure under dry conditions, we mounted a homemade drying chamber on the rheometer (see Fig. 2). It consists of an outer plexiglass chamber adapted on the bottom plate and an aluminum cover fixed on the top

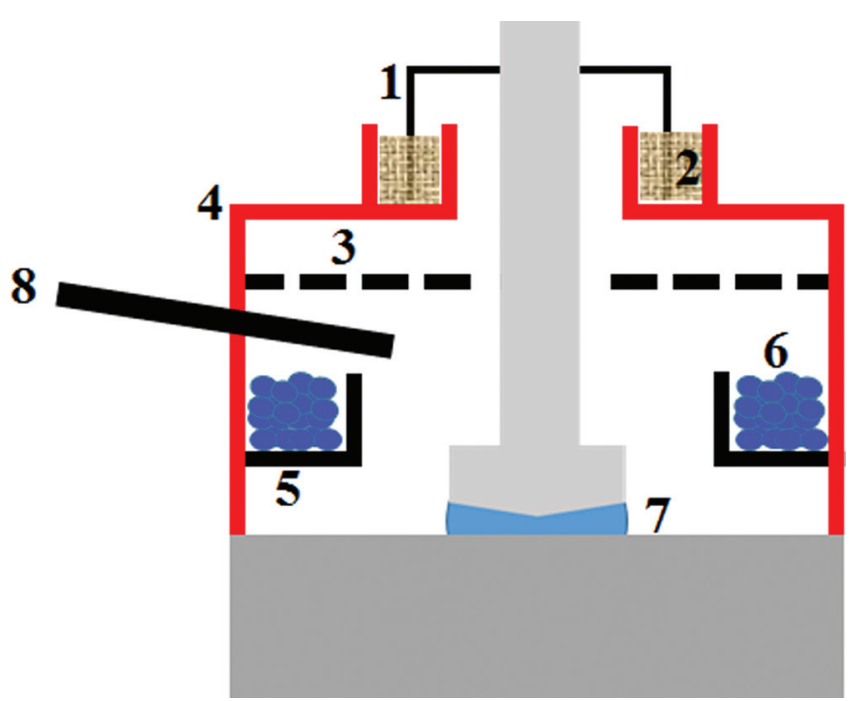

FIG. 2. Schematic illustration of the drying chamber: (1) Aluminum cover attached to the upper cone; (2) dodecane bath in order to completely seal the inner measurement region; (3) tissue soaked with dodecane to saturate the atmosphere; (4) outer plexiglass chamber adapted on the bottom Peltier plate; (5) inner chamber for the drying agent; (6) drying agent $\mathrm{P}_{2} \mathrm{O}_{5}$; (7) measured sample; and (8) humidity probe.

rotating rheometric fixture. These two elements were connected by means of a solvent (dodecane) bath which allowed rotation of the fixture and sealing of the measurement region. The inner atmosphere of the measured sample is kept dry by means of a drying agent (phosphorus pentoxide, $\mathrm{P}_{2} \mathrm{O}_{5}$ ). The relative humidity level was monitored by means of a highly sensitive (accuracy $\pm 1 \%$ ) humidity probe (Testo 645/445; Testo, Germany). On the other hand, measurements under humid conditions were carried out in the open air without any further precautions. Measurements under both humid (open air) and dry (using the drying chamber) conditions were conducted with samples prepared according to protocols 1 and 2 .

The quality of the results obtained with the drying chamber was ensured by taking all necessary precautions: No friction between elements 1 and 4 (they did not touch each other), keeping the viscosity of element 2 (dodecane) well below that of the lowest concentration investigated, and checking that element 4 (outer chamber) was firmly attached to the Peltier plate (dark gray part in Fig. 2) so as they rotated as one bulk unit.

\section{Karl-Fischer titration experiments}

In order to quantify the amount of water present in the final solutions, we used the well-known reference method called "Karl Fischer (KF) titration" [35]. A C20 coulometric KF Titrator (Mettler Toledo) was used with a Hydranal Coulomat E (Fluka) reagent for Karl Fischer titration.

\section{RESULTS AND DISCUSSION}

\section{A. Viscoelastic properties under humid conditions: Protocol-1}

Figure 3(a) shows the results of dynamic frequency sweeps for $5 \mathrm{~g} / \mathrm{l}$ EHUT solution prepared according to protocol-1 and measured immediately after preparation 

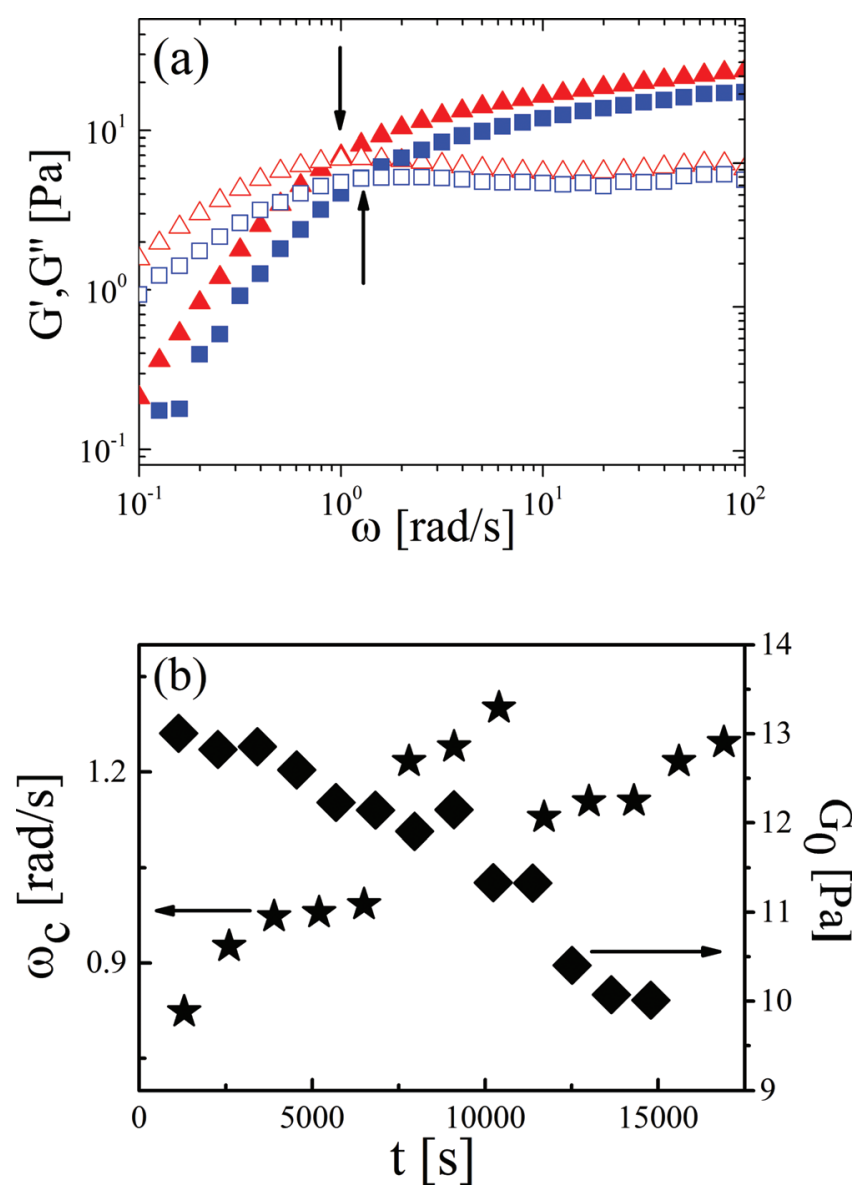

FIG. 3. (a) Frequency dependence of the elastic $\left(\mathrm{G}^{\prime}\right.$, filled symbols $)$ and loss $\left(\mathrm{G}^{\prime \prime}\right.$, open symbols) moduli for EHUT in dodecane at $\mathrm{C}=5 \mathrm{~g} / \mathrm{l}$ : Data are shown for the 1st test in open air (triangles) and a test after $5 \mathrm{~h}$ of repeated measurements (squares). No drying precautions were made in the preparation protocol neither during the measurement. Each measurement lasts about 20 min. (b) The evolution of $\mathrm{G}^{\prime}-\mathrm{G}^{\prime \prime}$ crossover frequency, $\omega_{c}$, (stars) and the plateau modulus $G_{0}$ (diamonds), during 5 h of measurements.

(triangles) and $5 \mathrm{~h}$ later (squares), in open air. We clearly observe a big difference between the two results. Indeed, after only $5 \mathrm{~h}$, the terminal relaxation is shifted to higher frequencies, whereas the plateau modulus decreases slightly.

Figure 3(b) depicts the evolution of the crossover frequency $\left(\omega_{c}\right)$ and the effective plateau modulus $\left(G_{0}\right)$ [36], as functions of time during $5 \mathrm{~h}$ of consecutive measurements. The increase in the crossover frequency by more than $50 \%$ and the concomitant decrease in the plateau modulus by about $20 \%$ corroborate the weakening of the viscoelastic behavior of the system. We note that the time dependence of the crossover frequency is nonmonotonic. It exhibits a maximum at about $10^{5} \mathrm{~s}$. This is considered as an apparent behavior and may relate in part to the fact that the ambient laboratory temperature can fluctuate much over the duration of the experiment, affecting the temperature inside the humidity control cell, hence the relative humidity. At any rate, this does not affect the message of the work.

\section{B. Assessment of the humidity control setup}

As mentioned above, in order to study the EHUT solution in dry atmosphere, the humidity was controlled by means of the drying chamber of Fig. 2. In this way, we managed to reduce the humidity of the sample environment substantially, as shown in Fig. 4, where we present the resulting humidity level as function of time under different experimental conditions, i.e., by sealing the system using the aluminum cover (element 1 in Fig. 2) and by removing this cover, hence exposing the sample to ambient conditions. The latter test was made in order to ensure that even if the drying chamber were not completely isolated, we would still have a fairly dry atmosphere around the measured sample (see Fig. 4).

As can be seen in Fig. 4, we can reach a very low humidity level, less than 5\% (region 3) when the drying chamber is closed, for approximately $16 \mathrm{~h}$, while the ambient relative humidity is about $40 \%$. Region 3 represents the dry experimental conditions referred to in this work. To further appreciate the efficiency of our drying chamber, we measured the humidity level before the top cover was inserted (region 2 in Fig. 4), which was stabilized to 5\%. Further, following $16 \mathrm{~h}$ of dry conditions, we removed the aluminum cover and recorded the evolution of the relative humidity for more than 3 days. After a first jump to 5\%, the humidity level increased gradually to $12.5 \%$ at the end of the measurement. This increase above $5 \%$ is due to the exposure of the sample environment to ambient humidity and the partial reaction of the drying agent with water vapor. However, this is a very slow process, which confirms the good control achieved in the present setup during measurements and handing.

For the present supramolecular system, humidity could in principle affect the EHUT (in powder before preparation and in solution) and the solvent. However, water has a very low solubility in dodecane [37,38]; hence, the effect is almost exclusively associated with EHUT. The sample treatment protocol which involves drying the EHUT powder for about $18 \mathrm{~h}$ using phosphorus pentoxide $\left(\mathrm{P}_{2} \mathrm{O}_{5}\right)$ helps in removing

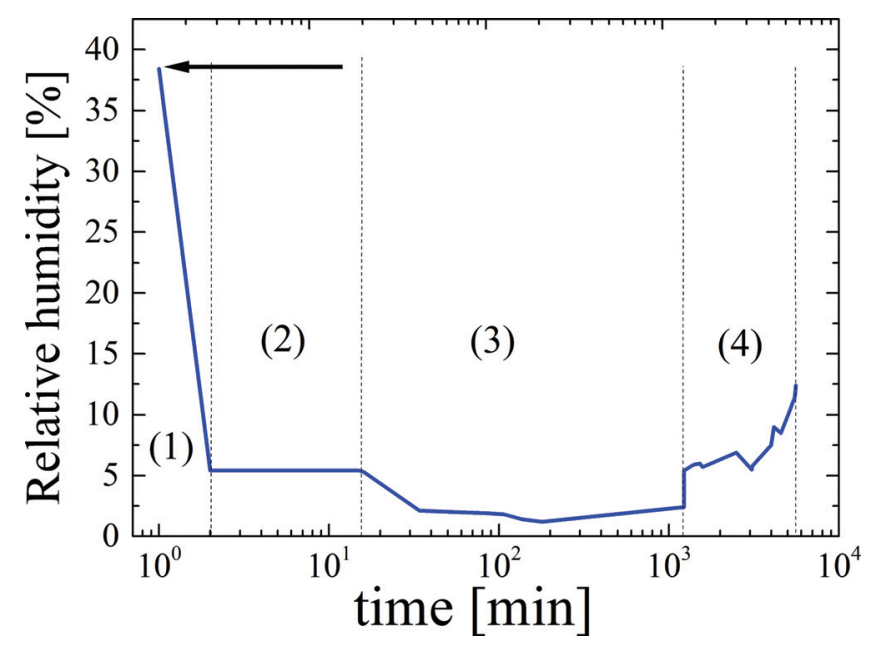

FIG. 4. Evolution of relative humidity at $25^{\circ} \mathrm{C}$ in different conditions: The arrow shows the ambient humidity outside the chamber. Region (1): Relative humidity measured by introducing the probe inside the drying chamber without closing the top with the aluminum cover (element 1 in Fig. 2). Region (2): Stabilization of the humidity level at 5\% inside the drying chamber without closing the top. Region (3): Relative humidity inside the sealed measurement area (by closing the top), reaching an average value of about $1.5 \%$. Region (4): Subsequent monitoring of the relative humidity inside the chamber after removing the top cover; its value jumped to $5 \%$ and kept increasing till about $12.5 \%$ at the end of the measurement. 
any humidity left in the solution and keeps the environment of the sample at a very low humidity level ( $<5 \%)$ (Fig. 4). We note that several works addressed the humidity effects in different systems and presented alternative setups for controlling it [39-44]. Here, by using the particular drying agent instead of only saturating the sample environment with a mixture of dry gas/wet gas, which is commonly used, especially in melts, we achieve a long-time control for the solutions. Humidity is continuously absorbed from the sample and kept at very low levels. For the specific setup used, the water content in air is estimated to be less than the $3.6 \mu \mathrm{g} / \mathrm{l}$, a cited value measured under flow conditions [45]. Given the large excess of $\mathrm{P}_{2} \mathrm{O}_{5}(6 \mathrm{~g})$ contained in the drying chamber, this estimation ensures the efficiency of our setup.

\section{Linear viscoelastic properties under dry conditions: Protocol-2}

We performed a sequence of dynamic frequency sweeps at three different experimental conditions for five concentrations prepared according to protocol-2 (see Sec. II A). The different concentrations probed are as follows: $2,2.75,4.88,8.41$, and $12 \mathrm{~g} / \mathrm{l}$. The time evolution of the $G^{\prime}-G^{\prime \prime}$ crossover frequency and the plateau modulus are reported in Figs. 5(a) and 5(b), respectively. First, we measured the freshly prepared sample in open air, and the respective data are referred to as humid-1. We then performed long measurements for about $24 \mathrm{~h}$ under dry conditions (the level of relative humidity was kept below $5 \%$ ), and the data are referred to as dry. Finally, the last measurements were performed after removing the drying chamber, with the data being referred to as humid- 2 .

The terminal crossover frequency decreases noticeably (by almost a factor of 3 ) when the humidity is removed and reaches a plateau at around $300 \mathrm{~min}$ [Fig. 5(a)]. The value of the crossover frequency remains virtually constant over almost $16 \mathrm{~h}$ of Dry condition measurements. We also observe that the terminal frequency at low concentrations depends weakly on time, in contrast to higher concentrations. Although this effect can be considered as insignificant, within experimental error, we note that even very low levels of humidity may affect the lower concentrations more than the higher ones.

On the other hand, upon re-exposing the measured sample to the air, the terminal frequency departs from its constant value. However, the final value of terminal frequency in humid-2 condition remains well below the initial one (humid-1). Surprisingly, this change in the terminal crossover frequency at three experimental conditions was not accompanied by any change in the plateau modulus. In fact, as shown in Fig. 5(b), $G_{O}$ remained virtually constant. This situation differs from that of Fig. 3(a) with respect to the sample preparation protocol: Unlike Fig. 3(a), here the samples in humid-1 condition were prepared from a predried powder according to protocol-2 (Sec. II A).

In order to appreciate the effects of humidity on the entire viscoelastic spectrum of EHUT solutions in dodecane, we show in Fig. 6(a) an example of the evolution of the frequencydependent $\mathrm{G}^{\prime}$ and $\mathrm{G}^{\prime \prime}$ at $\mathrm{C}=4.88 \mathrm{~g} / \mathrm{l}$ prepared according to protocol-2 (predried powder). Here, we compare the results obtained initially in open air (humid-1) and the results obtained
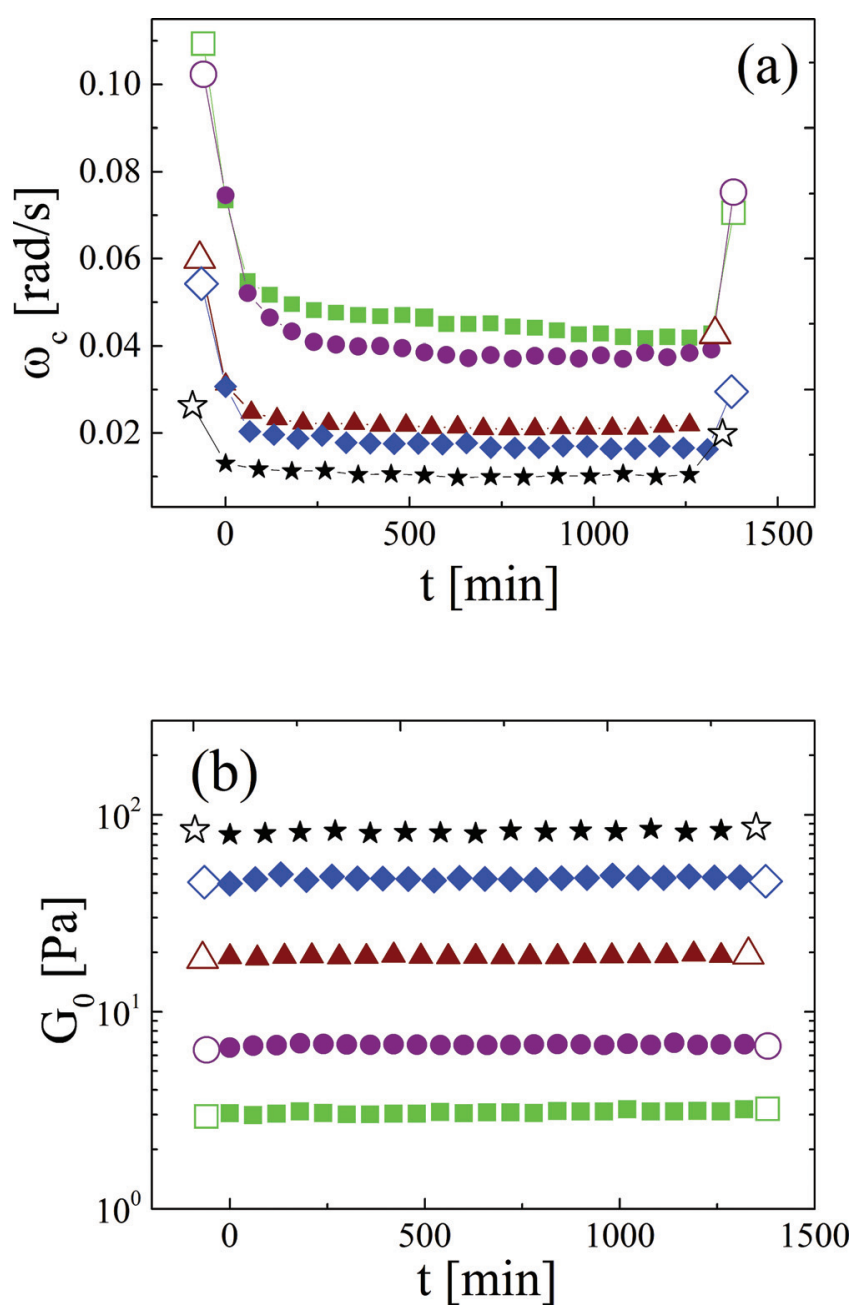

FIG. 5. Time evolution of (a) the terminal $G^{\prime}-G^{\prime \prime}$ crossover frequency and (b) the plateau modulus. Inset: The frequency at the minimum of $\mathrm{G}^{\prime \prime}, \omega_{\mathrm{b}}$, where we took the breaking time. Data are shown for five concentrations: $2 \mathrm{~g} / \mathrm{l}$ (squares), $2.75 \mathrm{~g} / \mathrm{l}$ (circles), $4.88 \mathrm{~g} / \mathrm{l}$ (triangles), $8.41 \mathrm{~g} / \mathrm{l}$ (diamonds), and $12 \mathrm{~g} / \mathrm{l}$ (stars). First open symbols: Humid-1 condition; close symbols: Dry condition; last open symbols: Humid-2 condition.

at different times under dry conditions (350, 840, and $1260 \mathrm{~min}$ ) with the data of a $5 \mathrm{~g} / \mathrm{l}$ sample prepared according to protocol-1. Contrary to the results obtained in the presence of humidity [Fig. 3(a)], we now manage to stabilize the system over $16 \mathrm{~h}$ of measurements. Although both samples have virtually the same concentration, the terminal relaxation time from the first experiment performed in open air for the sample prepared with protocol-2 is much slower compared to the one of the sample prepared with protocol-1. Furthermore, we note a weak increase in the value of the plateau modulus (determined at the frequency position where $G^{\prime \prime}$ has a minimum) from humid-1 to dry conditions for the sample prepared from a predried powder. However, the plateau modulus of the sample prepared from a non-pre-dried powder is noticeably different from the other data sets.

In Fig. 6(b), we compare two viscoelastic spectra in different conditions: Dry (1260 min) and humid-2 for the sample prepared from predried EHUT powder (protocol-2). A jump of the terminal frequency crossover from 0.02 to $0.04 \mathrm{rad} / \mathrm{s}$ is evident, without accompanied change in the plateau modulus. 

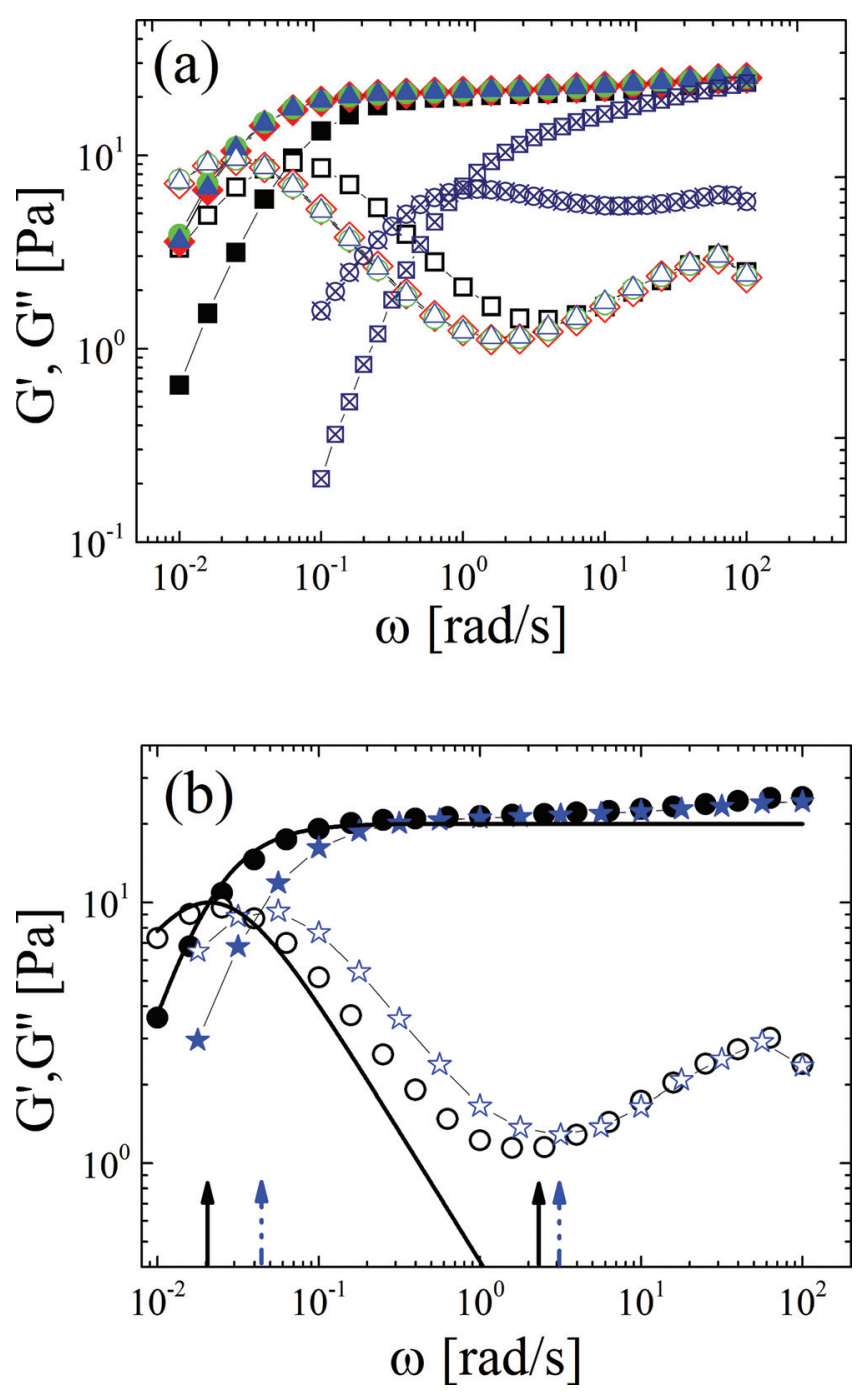

FIG. 6. Frequency dependence of the storage $\left(\mathrm{G}^{\prime}\right.$, filled symbols) and loss $\left(\mathrm{G}^{\prime \prime}\right.$, open symbols) moduli at different experimental conditions for EHUT in dodecane solution at $\mathrm{C}=4.88 \mathrm{~g} / \mathrm{l}$. (a) Humid-1 condition (squares) and Dry condition at $350 \mathrm{~min}$ (triangles), $840 \mathrm{~min}$ (diamonds), and $1260 \mathrm{~min}$ (circles). The Dry condition spectra appear with a low-frequency crossover of moduli at $\omega_{c}=0.021 \mathrm{rad} / \mathrm{s}$ [low frequency solid arrow in (b)] and $G_{0}=20 \mathrm{~Pa}$. Data are also shown for the 1 st test in open air with the $5 \mathrm{~g} / \mathrm{l}$ sample prepared according to protocol-1: $\left(\mathrm{G}^{\prime}\right.$, crossed squares) and $\left(\mathrm{G}^{\prime \prime}\right.$, crossed circles). (b) Dry condition at 1260 min (circles), respective data for humid-2 condition, after removing the cover (stars), showing an immediate increase in $\omega_{c}$ to $0.042 \mathrm{rad} / \mathrm{s}$ (low-frequency dashed arrow), but no change in the plateau modulus. The high-frequency solid and dashed arrows show the small difference between the frequencies at the minimum of $\mathrm{G}^{\prime \prime}$ for the dry and humid-2 data, respectively. The solid lines indicate the fit of dry condition data with the Maxwell.

Given that the terminal relaxation time of the EHUT solution is a function of $\tau_{r e p}$ and $\tau_{b}$ as discussed in Sec. I [7], we highlight in Fig. 6(b) the much smaller effect of the humidity on the breaking time $\left(\tau_{b}=1 / \omega_{b}\right)[27,28]$ compared to that on the terminal relaxation time. The choice of the frequency corresponding to the minimum of $\mathrm{G}^{\prime \prime}$ as an estimate of the inverse of the breaking time (in fact, the end of the breaking process) is consistent with the literature and further motivated by the fact that it clearly deviates from the Maxwell model (Fig. 6) and also corresponds to the deviation of the Cole-Cole plot from the semispherical shape. [7-9,12,28,32]. In this context, it should be mentioned that different methods to estimate the average breaking time have been reported in the literature. Shikata et al. in [12] took the breaking time as the inverse of the frequency at the high-frequency maximum in $G^{\prime \prime}$. This is a good estimation of the average breaking time; however, the high-frequency maximum is not always reached experimentally [see, for example, Fig. 3(a)]. On the other hand, the minimum of $G^{\prime \prime}$ will yield a value associated with the maximum estimated time in relation to chain-breaking process. Nevertheless, by considering the maximum of $G^{\prime \prime}$, no effect of humidity is detected at different experimental conditions (Fig. 6). Turner and Cates proposed another method to estimate the breaking time from the linear data using the Cole-Cole representation [46]. However, this method sometimes yields unphysically high values of the breaking times in some supramolecular systems and seems to be relevant particularly for lower concentrations. It is therefore beyond any doubt that the changes in terminal relaxation time $\left(\tau_{t}\right)$ are mainly due to the changes in the reptation time rather than the breaking time.

\section{Nonlinear response under dry conditions: A comparison between protocol-1 and protocol-2}

We also checked the humidity effect on the nonlinear rheology by performing shear stress start-up experiments with a sample of concentration of $5 \mathrm{~g} / \mathrm{l}$ prepared with both protocol-1 (no powder drying prior to preparation) and protocol-2 (with powder dried overnight at $50{ }^{\circ} \mathrm{C}$ prior to preparation). In Fig. 7(a), we present the results for the transient shear viscosity at different rates for the sample prepared with protocol-1 and measured directly after the end of preparation without any drying on the rheometer. Also shown are results for the sample prepared with protocol-2 and measured in the dry condition (Sec. III C). It is evident that the transient viscosity under protocol2 is larger than that under protocol-1. We also highlight the weaker overshoot in protocol-1 as compared to protocol-2, and, in particular, it is the fact that at $0.1 \mathrm{~s}^{-1}$, there is no overshoot for protocol-1. It is also worth noting that at shear rates of $3 \mathrm{~s}^{-1}$ and higher, a transient shear-hardening is observed, being more pronounced for the dry samples (with longer chains). This has been reported for associating systems and attributed to stretching [47]. The nonlinear response EHUT assemblies are complex [21], and a detailed analysis of the present systems will be presented in a future study.

Figure 7(b) shows the viscosity at the overshoot as function of shear rate for the two cases studied. We observe larger viscosity values for the sample measured in the Dry condition (protocol-2). This difference is reduced at higher shear rates, apparently due to flow-induced effects, but this discussion is beyond the scope of the present work.

\section{E. Rationalization of the role of humidity}

The softening of the viscoelastic behavior of the EHUT supramolecular structure observed in the rheological data discussed in Sec. III B is consistent with the recent results of Francisco et al. in [14]. These authors reported the effects of chain stoppers on the viscoelastic proprieties of EHUT. Different chain stoppers were used, having the ability to interact with EHUT monomers via hydrogen bonds: Benzyl 

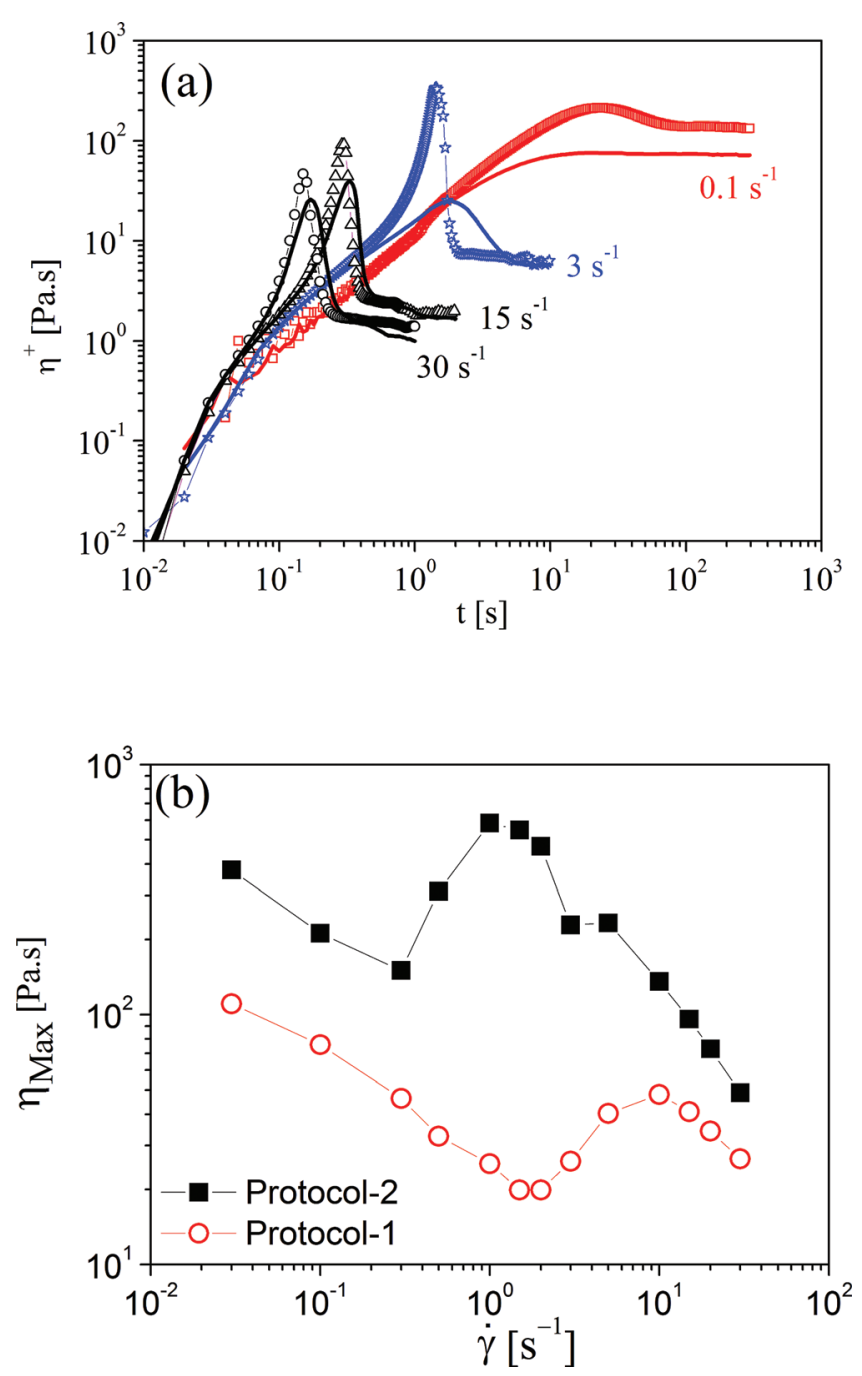

FIG. 7. (a) Transient viscosity versus time at shear rates (indicated in the plot) for a sample at $\mathrm{C}=5 \mathrm{~g} / \mathrm{l}$ prepared according to protocol-1 (lines) and measured without any further drying (solid lines) and for a sample at the same concentration $(5 \mathrm{~g} / \mathrm{l})$ prepared according to protocol-2 (symbols) and measured in the Dry condition (symbols-thin lines). (b) The maximum viscosity at the overshoot peak versus shear rate for the two samples prepared and measured as described above (legends are inside the plot).

alcohol and ethanol. It was observed that adding a very small amount of ethanol molecules (at equimolar ratio EHUT/ethanol) resulted in a substantial drop of the relaxation time by a factor of 2.38 and a rather modest drop of the plateau modulus by a factor of 1.15 . The crucial role of chain stoppers on the EHUT supramolecular structures was also identified. It leads to a weakening of their viscoelastic proprieties through the competition of chain stopper-EHUT hydrogen bonding and EHUT-EHUT self-association, which stops the growth of the supramolecular assembly, hence shortening its average chain length. It turns out that several studies have focused on the chain stopper effect on the rheological properties of the EHUT supramolecular assembly, supporting the above picture $[14,18,19,48]$. Inspired by these findings, we propose that in the presence of humidity, water molecules can act as chain stoppers, i.e., the water-EHUT hydrogen bonding outbalances the EHUT-EHUT self-association. Consequently, the average length of the supramolecular EHUT chains is shortened and their viscoelastic properties are weakened.
Indeed, the shortening of the supramolecular chains should be responsible of the faster relaxation observed in open air [Figs. 5(a) and 6(a)]. Note that the same effect was highlighted in the results presented in Fig. 3, where the terminal crossover frequency shifted toward higher values in open air. Concomitantly, the weak (albeit non-negligible) drop of plateau modulus observed in Fig. 3(a) is attributed to the fact that the EHUT/dodecane solution was prepared and measured without any drying precautions. Water appears responsible for a reduction in the average length of the EHUT micelle, accompanied by a possible increase in polydispersity of the supramolecular chains, which makes the constraint release effects more pronounced [29,30], hence leading to the observed decrease in the magnitude of the storage modulus and its stronger decrease with decreasing frequency [Figs. 3(a) and 6(a)]. The reduction in the micellar average length and polydispersity will also weaken the nonlinear viscoelastic response of the material. In fact, the longer the chains, the larger the maximum stress (or viscosity) they experience before it decreases to steady state (due to the apparent disentanglement and/or breaking), and the sharper the overshoot (see Fig. 7).

\section{F. Quantifying water content in EHUT solutions}

The water content in the final solutions under humid conditions in the samples prepared with protocol-2 was measured by means of the Karl-Fischer method. Despite the careful powder drying prior to the sample preparation, all solutions were found to have almost the same content of water, amounting to about $25 \mathrm{ppm}$ (Table I). This is due to hygroscopic nature of the EHUT assembly mentioned earlier. Note that it was not possible to measure the $12 \mathrm{~g} / \mathrm{l}$ solution due to its high viscosity.

In terms of molar concentrations, the above results lead to the values of the [Water]/[EHUT] ratio ranging from 1/3.35 to $1 / 15$, which are in the same range as the previous data reported for EHUT systems in the presence of chain stoppers $[14,18]$. We also calculated the exact mass of water that was present in the actual measured sample volume $(0.41 \mathrm{ml})$ and found a value of about $10 \mu \mathrm{g}$. This is admittedly a very low amount and is hardly detectable or measurable with conventional spectroscopic techniques such infrared spectroscopy for example.

In order to appreciate how consistent the water content measured with KF compared to the reduction in the terminal relaxation time by factor of 3 , we first estimate the direct proportionality between the relaxation time and the supramolecular chain length. Using Eq. (1) and the scaling of Cates for the breaking time, we end up with $\tau_{t} \sim L$, which suggests

TABLE I. Karl-Fischer titration results for the amount of water present in the EHUT solutions.

\begin{tabular}{lc}
\hline \hline $\mathrm{C}(\mathrm{g} / \mathrm{l})$ & Amount of water $(\mathrm{ppm})$ \\
\hline 2 & 25.7 \\
2.75 & 24.8 \\
4.88 & 27.7 \\
8.41 & 26.9 \\
\hline \hline
\end{tabular}


that a drop of the relaxation time by a factor of 3 implies a reduction of the length by factor of 3 . By using the chain stopper model [18], the water needed to achieve such a drop of the relaxation time amounts to about $10 \mathrm{ppm}$, based on the assumption that we need at least two water molecules to stop the growth of one chain. Considering that we typically need more than two water molecules to stop the growth of a chain, the KF results of Table I can be rationalized. Overall, these results strongly suggest that one should take extreme precautions for sample preparation and selection of measurement protocols in order to achieve truly dry conditions. They confirm that rheology is a particularly sensitive quantitative tool.

\section{G. Scaling viscoelasticity in dry conditions}

Figure 8(a) presents the terminal relaxation time as function of EHUT concentration under dry conditions (samples prepared with protocol-2). In the same figure, we plot the
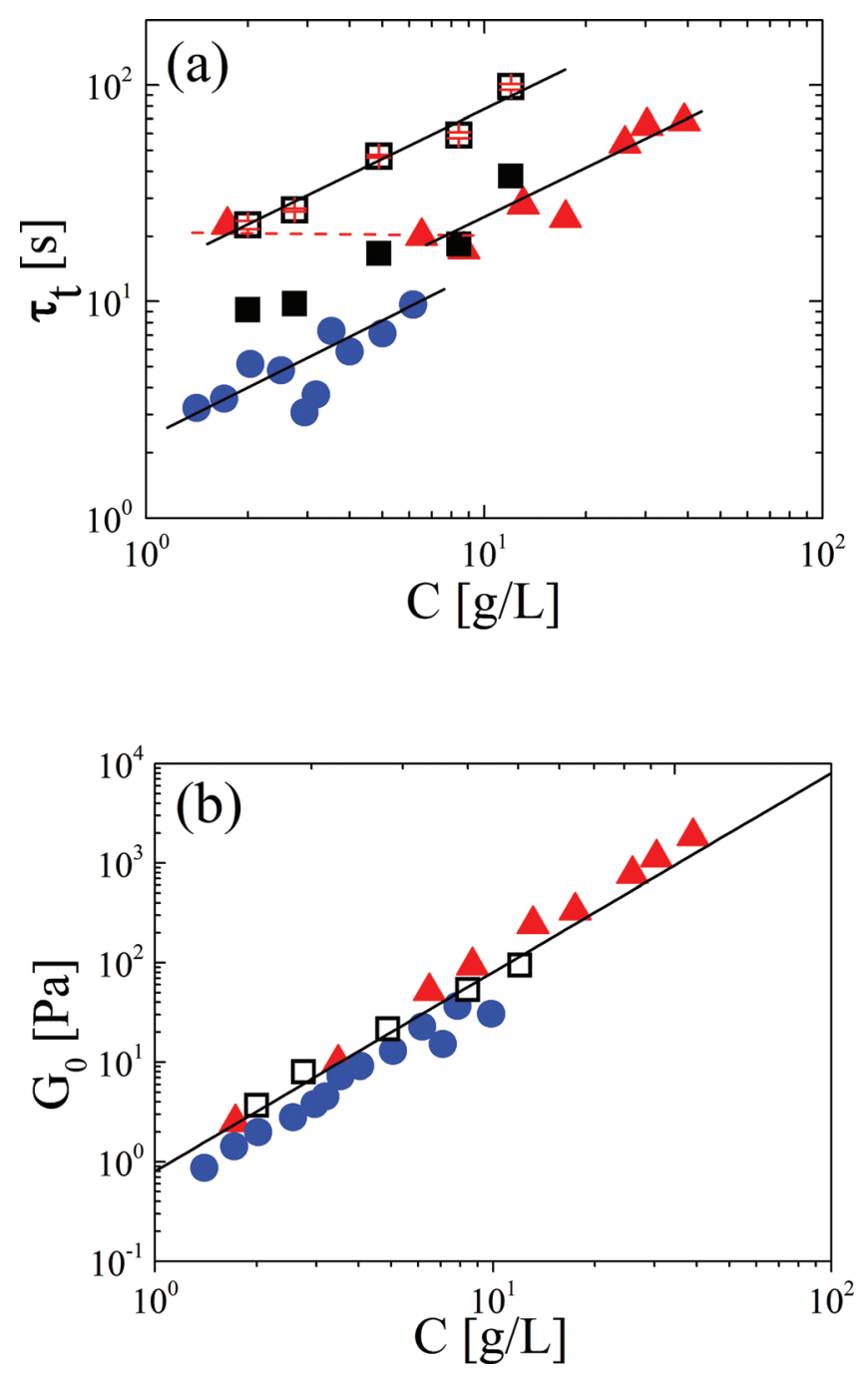

FIG. 8. (a) Experimental data of the terminal relaxation time versus concentration obtained using the humidity control setup. The different symbols refer to: Humid-1 (filled squares), dry (open squares), data from [5] (triangles), and data from [12] (circles). All solid lines have a slope of $0.77 \pm 0.06$, which is the extracted scaling exponent. The dotted horizontal line is drawn to guide the eye through the data from [12] (see text). (b) Respective data of the plateau modulus versus concentration. The line has a slope of $2.03 \pm 0.21$. data for initially humid systems (humid-1; protocol-2) as well as data from the literature without humidity control $[5,12]$. It is evident that under dry conditions the terminal relaxation times reach the highest values, while the data at different concentrations appear consistent without appreciable scattering. On the other hand, data suffering from humidity at different levels exhibit pronounced scattering and even change scaling behavior with concentration [5]. This seems to corroborate the above proposed idea about the effect of humidity on the terminal relaxation time. We therefore attribute that discrepancies among different datasets for the same EHUT system to the variable, uncontrolled level of humidity $[5,12]$. Furthermore, we suggest that the observed concentration independence of the relaxation time at low concentrations in [12] reflects the strong chain stopper effect of water. Actually, a plateau in the relaxation time was also observed by Knoben et al. in [19] in the same range of concentrations $(1-10 \mathrm{~g} / \mathrm{l})$, by introducing a small fraction $(0.015)$ of $[2,4-$ bis(dibutylureido)toluene, DBUT]. The latter is an efficient chain stopper for the EHUT supramolecular chains reported, because it has the ability for simultaneous formation of four hydrogen bonds with EHUT without having the possibility to bond with another DBUT or EHUT monomer, hence stopping the growth of the chain.

Interestingly, aside for the difference in the magnitude of the terminal relaxation time, its concentration dependence follows a universal scaling $\tau_{\mathrm{t}} \sim C^{0.77}$, with the exception of the lowest concentration in [12]. This disagrees with the scaling prediction in Eq. (2) of Cates and Candau [8]. We note that a disagreement of this experimental scaling with the prediction in Eq. (2) was found in many other living polymers, such as surfactant WLMs [32], and attributed to the different scaling of the average micellar length with concentration (which, in the theory of Cates scales as $L \sim C^{0.5}$ ). We tentatively attribute this difference to a polydispersity in the length of supramolecular EHUT chains, possibly exceeding their most probable value of 2 , which is considered in the model of Cates. The latter value is a direct consequence of the nonisodesmic nature of the EHUT self-association reported in [10]. The consequences of this kind of selfassembly mechanism are strong association, sharp transition between monomerlike and polymerlike properties and a polydispersity likely larger than 2 [8,10,49-52]. By invoking the constraint release ideas developed for polymers [30,53-55], in such polydisperse systems the short chains act as effective solvents of the longer ones, speeding up their relaxation. Given the living character of this system, chain polydispersity is expected to be different at different concentrations. In fact, knowing the distribution of chain lengths at different concentrations, one should be able to predict the terminal relaxation time, say by means of a relatively simple double reptation model $[55,56]$. Such a task represents a future direction in this research field. Note also that the relaxation of the fast chains and redistribution of entanglements of the longer ones may affect the breaking time as well (see Sec. III C).

In contrast to the relaxation time, we observe in Fig. 8(b) that the concentration scaling of the plateau modulus is consistent with the prediction in Eq. (3), with all experiments 
indicating that $G_{0} \sim C^{2.03 \pm 0.21}$. In this figure, the data from [5] are slightly below the other ones, as expected, given the weak humidity effect on the plateau modulus [Fig. 3(a)].

\section{CONCLUSION}

The linear and nonlinear viscoelasticity of EHUT, a supramolecular organogelator formed by hydrogen bonding association of a bifunctional monomer in apolar solvent dodecane, is found to be strongly affected by the presence of humidity. Even in small amounts, water molecules compete with the EHUT-EHUT bonding; hence, a fraction of EHUT monomers forms hydrogen bonds with water instead of other EHUT. This eventually yields a decrease in the average chain length, possibly weak increase in polydispersity and speeds up the terminal relaxation time. At the same time, the plateau modulus decreases only slightly, in the presence of substantial humidity. Similarly, nonlinear rheology is also strongly affected by the presence of humidity, which is responsible of the weakening of the transient response of the material during start-up shear experiments. Careful sample preparation protocol and measurements under dry conditions proved necessary in order to clarify this important issue and resolve discrepancies in published experimental data with the same system. Hence, achieving truly dry conditions is necessary for investigating this type of supramolecular assemblies having the propensity to bind with water molecules. Our data over a reasonably wide range of concentrations in the tube regime of the phase diagram of the EHUT/ dodecane system reveal the correct experimental scaling laws. The plateau modulus scales essentially with the square of the concentration, in a very good agreement with the theoretical predictions. On the other hand, the terminal relaxation time exhibits a much weaker scaling with an exponent of 0.77 , quite smaller than the theoretical prediction of 1.25 . We tentatively attribute this difference to the polydispersity in chain length of these supramolecular living polymers, which introduces constraint release effects. Accounting for the length distribution and its consequences on both breaking and terminal relaxation times is a challenge for the future.

\section{ACKNOWLEDGMENTS}

The authors acknowledge the support from the EU (Marie Sklodowska Curie ITN Supolen, GA 607937).

\section{References}

[1] Terech, P., and R. G. Weiss, "Low molecular mass gelators of organic liquids and the properties of their gels," Chem. Rev. 97, 3133-3160 (1997).

[2] George, M., and R. G. Weiss, "Molecular organogels. Soft matter comprised of low-molecular-mass organic gelators and organic liquids," Acc. Chem. Res. 39, 489-497 (2006).

[3] Dastidar, P., "Supramolecular gelling agents: Can they be designed?," Chem. Soc. Rev. 37, 2699-2715 (2008).

[4] Weiss, R. G., "The past, present, and future of molecular gels. What is the status of the field, and where is it going?," J. Am. Chem. Soc. 136, 7519-7530 (2014).
[5] Ducouret, G., C. Chassenieux, S. Martins, F. Lequeux, and L. Bouteiller, "Rheological characterisation of bis-urea based viscoelastic solutions in an apolar solvent," J. Colloid Interface Sci. 310, 624-629 (2007).

[6] Lortie, F., S. Boileau, L. Bouteiller, C. Chassenieux, B. Demé, G. Ducouret, M. Jalabert, F. Lauprêtre, and P. Terech, "Structural and rheological study of a bis-urea based reversible polymer in an apolar solvent," Langmuir 18, 7218-7222 (2002).

[7] Cates, M., "Reptation of living polymers: Dynamics of entangled polymers in the presence of reversible chain-scission reactions," Macromolecules 20, 2289-2296 (1987).

[8] Cates, M., and S. Candau, "Statics and dynamics of worm-like surfactant micelles," J. Phys.: Condens. Matter 2, 6869-6892 (1990).

[9] Berret, J. F., J. Appell, and G. Porte, "Linear rheology of entangled wormlike micelles," Langmuir 9, 2851-2854 (1993).

[10] Simic, V., L. Bouteiller, and M. Jalabert, "Highly cooperative formation of bis-urea based supramolecular polymers," J. Am. Chem. Soc. 125, 13148-13154 (2003).

[11] Bellot, M., and L. Bouteiller, "Thermodynamic description of bis-urea self-assembly: Competition between two supramolecular polymers," Langmuir 24, 14176-14182 (2008).

[12] Shikata, T., T. Nishida, B. Isare, M. Linares, R. Lazzaroni, and L. Bouteiller, "Structure and dynamics of a bisurea-based supramolecular polymer in n-dodecane," J. Phys. Chem. B 112, 8459-8465 (2008).

[13] Sabadini, E., K. R. Francisco, and L. Bouteiller, "Bis-urea-based supramolecular polymer: The first self-assembled drag reducer for hydrocarbon solvents," Langmuir 26, 1482-1486 (2009).

[14] Francisco, K. R., C. A. Dreiss, L. Bouteiller, and E. Sabadini, "Tuning the viscoelastic properties of bis (urea)-based supramolecular polymer solutions by adding cosolutes," Langmuir 28, 14531-14539 (2012).

[15] Callies, X., C. Fonteneau, C. Véchambre, S. Pensec, J.-M. Chenal, L. Chazeau, L. Bouteiller, G. Ducouret, and C. Creton, "Linear rheology of bis-urea functionalized supramolecular poly (butylacrylate) s: Part I-weak stickers," Polymer 69, 233-240 (2015).

[16] Isare, B., S. Pensec, M. Raynal, and L. Bouteiller, "Bisurea-based supramolecular polymers: From structure to properties," C. R. Chim. 19, 148-156 (2016).

[17] Van der Gucht, J., N. Besseling, W. Knoben, L. Bouteiller, and M. C. Stuart, "Brownian particles in supramolecular polymer solutions," Phys. Rev. E 67, 051106 (2003).

[18] Lortie, F., S. Boileau, L. Bouteiller, C. Chassenieux, and F. Lauprêtre, "Chain stopper-assisted characterization of supramolecular polymers," Macromolecules 38, 5283-5287 (2005).

[19] Knoben, W., N. Besseling, L. Bouteiller, and M. C. Stuart, "Dynamics of reversible supramolecular polymers: Independent determination of the dependence of linear viscoelasticity on concentration and chain length by using chain stoppers," Phys. Chem. Chem. Phys. 7, 2390-2398 (2005).

[20] Pinault, T., B. Isare, and L. Bouteiller, "Solvents with similar bulk properties induce distinct supramolecular architectures," ChemPhysChem 7, 816-819 (2006).

[21] Van der Gucht, J., M. Lemmers, W. Knoben, N. Besseling, and M. Lettinga, "Multiple shear-banding transitions in a supramolecular polymer solution," Phys. Rev. Lett. 97, 108301 (2006).

[22] Jacob, A. R., A. P. Deshpande, and L. Bouteiller, "Large amplitude oscillatory shear of supramolecular materials," J. Non-Newtonian Fluid Mech. 206, 40-56 (2014).

[23] Lescanne, M., P. Grondin, A. d'Aléo, F. Fages, J.-L. Pozzo, O. M. Monval, P. Reinheimer, and A. Colin, "Thixotropic organogels based on a simple N-hydroxyalkyl amide: Rheological and aging properties," Langmuir 20, 3032-3041 (2004). 
[24] Lescanne, M., A. Colin, O. Mondain-Monval, F. Fages, and J.-L. Pozzo, "Structural aspects of the gelation process observed with low molecular mass organogelators," Langmuir 19, 2013-2020 (2003).

[25] Schmidt, R., M. Schmutz, M. Michel, G. Decher, and P. J. Mésini, "Organogelation properties of a series of oligoamides," Langmuir 18, 5668-5672 (2002).

[26] Cates, M., "Dynamics of living polymers and flexible surfactant micelles: Scaling laws for dilution,” J Phys. 49, 1593-1600 (1988).

[27] Rogers, S. A., M. A. Calabrese, and N. J. Wagner, "Rheology of branched wormlike micelles," Curr. Opin. Colloid Interface Sci. 19, 530-535 (2014).

[28] Kim, S., J. Mewis, C. Clasen, and J. Vermant, "Superposition rheometry of a wormlike micellar fluid," Rheol. Acta 52, 727-740 (2013).

[29] Doi, M., and S. Edwards, The Theory of Polymer Dynamics (Claredon, Oxford, 1986).

[30] Colby, R., and M. Rubinstein, Polymer Physics (Oxford University, New York, 2003), pp. 274-281.

[31] Larson, R. G., "The lengths of thread-like micelles inferred from rheology," J. Rheol. 56, 1363-1374 (2012).

[32] Berret, J.-F., "Rheology of wormlike micelles: Equilibrium properties and shear banding transitions," in Molecular Gels (Springer, Dordrecht, The Netherlands, 2006), Vol. 19, pp. 667-720.

[33] Soltero, J., J. Puig, and O. Manero, "Rheology of the cetyltrimethylammonium tosilate-water system. 2. Linear viscoelastic regime," Langmuir 12, 2654-2662 (1996).

[34] Shchipunov, Y. A., and H. Hoffmann, "Growth, branching, and local ordering of lecithin polymer-like micelles," Langmuir 14, 6350-6360 (1998).

[35] Fischer, K., "Neues Verfahren zur maßanalytischen Bestimmung des Wassergehaltes von Flüssigkeiten und festen Körpern," Angew. Chem. 48, 394-396 (1935).

[36] Liu, C., J. He, E. Van Ruymbeke, R. Keunings, and C. Bailly, "Evaluation of different methods for the determination of the plateau modulus and the entanglement molecular weight," Polymer 47, 4461-4479 (2006).

[37] Schatzberg, P., "Solubilities of water in several normal alkanes from C7 to C161," J. Phys. Chem. 67, 776-779 (1963).

[38] Shaw, D. G., A. Maczynski, M. Goral, B. Wisniewska-Goclowska, A. Skrzecz, I. Owczarek, K. Blazej, M.-C. Haulait-Pirson, G. T. Hefter, and F. Kapuku, "IUPAC-NIST solubility data series. 81. Hydrocarbons with water and seawater-revised and updated. Part 10. C11 and C12 hydrocarbons with water," J. Phys. Chem. Ref. Data 35, 153-203 (2006)

[39] Caire, B. R., M. A. Vandiver, and M. W. Liberatore, "Mechanical testing of small, thin samples in a humidity-controlled oven," Rheol. Acta 54, 253-261 (2015).

[40] Majsztrik, P., A. Bocarsly, and J. Benziger, "An instrument for environmental control of vapor pressure and temperature for tensile creep and other mechanical property measurements," Rev. Sci. Instrum. 78, 103904 (2007).
[41] White, C. C., D. L. Hunston, K. T. Tan, J. Hettenhouser, and J. D. Garver, "An accelerated exposure and testing apparatus for building joint sealants,” Rev. Sci. Instrum. 84, 095113 (2013).

[42] Vandiver, M. A., B. R. Caire, J. R. Carver, K. Waldrop, M. R. Hibbs, J. R. Varcoe, A. M. Herring, and M. W. Liberatore, "Mechanical characterization of anion exchange membranes by extensional rheology under controlled hydration,” J. Electrochem. Soc. 161, H677-H683 (2014).

[43] Liu, Y., J. L. Horan, G. J. Schlichting, B. R. Caire, M. W. Liberatore, S. J. Hamrock, G. M. Haugen, M. A. Yandrasits, S. N. Seifert, and A. M. Herring, "A small-angle X-ray scattering study of the development of morphology in films formed from the $3 \mathrm{M}$ perfluorinated sulfonic acid ionomer," Macromolecules 45, 7495-7503 (2012).

[44] Schlichting, G. J., J. L. Horan, J. D. Jessop, S. E. Nelson, S. N. Seifert, Y. Yang, and A. M. Herring, "A hybrid organic/inorganic ionomer from the copolymerization of vinylphosphonic acid and zirconium vinylphosphonate," Macromolecules 45, 3874-3882 (2012).

[45] Trusell, F., and H. Diehl, "Efficiency of Chemical Desiccants," Anal. Chem. 35, 674-677 (1963).

[46] Turner, M., and M. Cates, "Linear viscoelasticity of living polymers: A quantitative probe of chemical relaxation times," Langmuir 7, 1590-1594 (1991).

[47] Berret, J.-F., Y. Séréro, B. Wnkelman, D. Calvet, A. Collet, and M. Viguier, "Nonlinear rheology of telechelic polymer networks," J. Rheol. 45, 477-492 (2001).

[48] Pinault, T., C. Cannizzo, B. Andrioletti, G. Ducouret, F. Lequeux, and L. Bouteiller, "Anions as efficient chain stoppers for hydrogen-bonded supramolecular polymers," Langmuir 25, 8404-8407 (2009).

[49] De Greef, T. F., M. M. Smulders, M. Wolffs, A. P. Schenning, R. P. Sijbesma, and E. Meijer, "Supramolecular polymerization," Chem. Rev. 109, 5687-5754 (2009).

[50] Zhao, D., and J. S. Moore, "Nucleation-elongation: A mechanism for cooperative supramolecular polymerization," Org. Biomol. Chem. 1, 3471-3491 (2003).

[51] Wittmer, J., A. Milchev, and M. Cates, "Dynamical Monte Carlo study of equilibrium polymers: Static properties," J. Chem. Phys. 109, 834-845 (1998).

[52] Wittmer, J., P. van der Schoot, A. Milchev, and J. Barrat, "Dynamical Monte Carlo study of equilibrium polymers. II. The role of rings," J. Chem. Phys. 113, 6992-7005 (2000).

[53] Viovy, J. L., M. Rubinstein, and R. H. Colby, "Constraint release in polymer melts: Tube reorganization versus tube dilation," Macromolecules 24, 3587-3596 (1991).

[54] Tsenoglou, C., "Molecular weight polydispersity effects on the viscoelasticity of entangled linear polymers," Macromolecules 24, 1762-1767 (1991).

[55] Des Cloizeaux, J., "Double reptation vs. simple reptation in polymer melts," EPL (Europhys. Lett.) 5, 437-442 (1988).

[56] Van Ruymbeke, E., R. Keunings, V. Stephenne, A. Hagenaars, and C. Bailly, "Evaluation of reptation models for predicting the linear viscoelastic properties of entangled linear polymers," Macromolecules 35, 2689-2699 (2002). 